\title{
Speitkamp, Winfried
}

\section{Vom Bauhaus lernen: Das Staatliche Bauhaus von 1919 und die Universität der Zukunft}

Thole, Friederike [Hrsg.]; Wedde, Sarah [Hrsg.]; Kather, Alexander [Hrsg.]: Über die Notwendigkeit der Historischen Bildungsforschung. Wegbegleiter*innenschrift für Edith Glaser. Bad Heilbrunn : Verlag Julius Klinkhardt 2021, S. 161-171

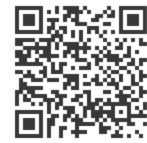

\section{Quellenangabe/ Citation:}

Speitkamp, Winfried: Vom Bauhaus lernen: Das Staatliche Bauhaus von 1919 und die Universität der Zukunft - In: Thole, Friederike [Hrsg.]; Wedde, Sarah [Hrsg.]; Kather, Alexander [Hrsg.]: Über die Notwendigkeit der Historischen Bildungsforschung. Wegbegleiter*innenschrift für Edith Glaser. Bad Heilbrunn : Verlag Julius Klinkhardt 2021, S. 161-171 - URN: urn:nbn:de:0111-pedocs-233458 - DOI: 10.25656/01:23345

\section{http://nbn-resolving.org/urn:nbn:de:0111-pedocs-233458}

http://dx.doi.org/10.25656/01:23345

in Kooperation mit / in cooperation with:

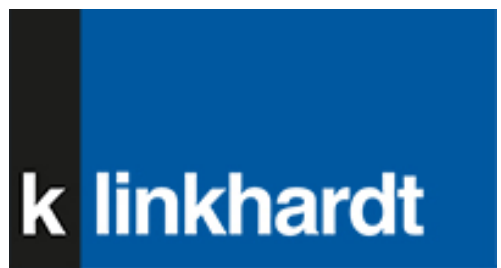

http://www.klinkhardt.de

\section{Nutzungsbedingungen}

Dieses Dokument steht unter folgender Creative Commons-Lizenz: http://creativecommons.org/licenses/by-nc-sa/4.0/deed.de - Sie dürfen das Werk bzw. den Inhalt unter folgenden Bedingungen vervielfältigen, verbreiten und öffentlich zugänglich machen sowie Abwandlungen und Bearbeitungen des Werkes bzw. Inhaltes anfertigen: Sie müssen den Namen des Autors/Rechteinhabers in der von ihm festgelegten Weise nennen. Dieses Werk bzw. der Inhalt darf nicht für kommerzielle Zwecke verwendet werden. Die neu entstandenen Werke bzw. Inhalte dürfen nur unter Verwendung von Lizenzbedingungen weitergegeben werden, die mit denen dieses Lizenzvertrages identisch oder vergleichbar sind.

Mit der Verwendung dieses Dokuments erkennen Sie die Nutzungsbedingungen an.

\section{Terms of use}

This document is published under following Creative Commons-License: http://creativecommons.org/licenses/by-nc-sa/4.0/deed.en - You may copy, distribute and transmit, adapt or exhibit the work in the public and alter, transform or change this work as long as you attribute the work in the manner specified by the author or licensor. You are not allowed to make commercial use of the work. If you alter, transform, or change this work in any way, you may distribute the resulting work only under this or a comparable license.

By using this particular document, you accept the above-stated conditions of use.

\section{Kontakt / Contact:}

\section{peDOCs}

DIPF | Leibniz-Institut für Bildungsforschung und Bildungsinformation Informationszentrum (IZ) Bildung

E-Mail: pedocs@dipf.de

Internet: www.pedocs.de

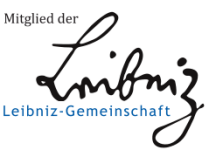




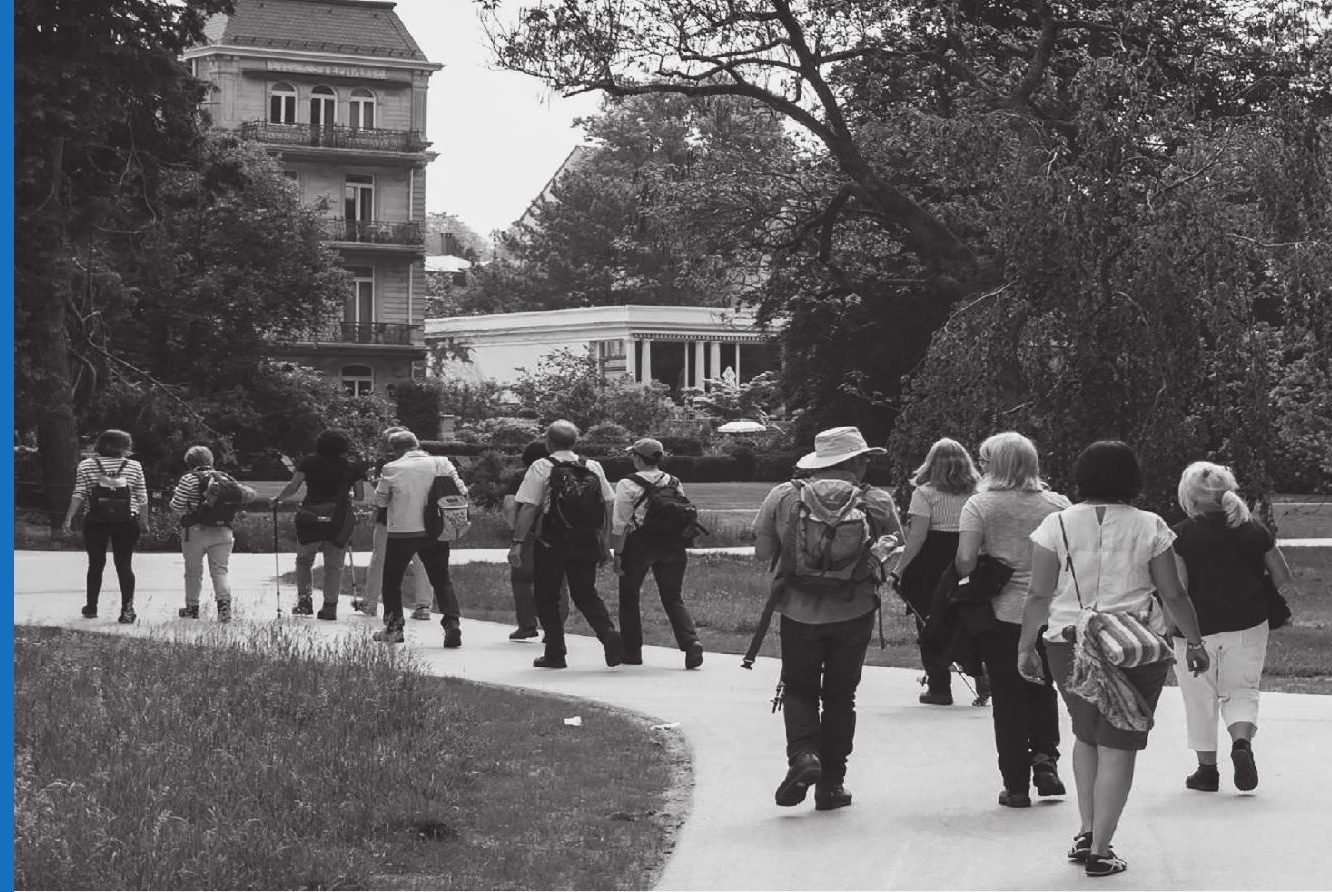

Friederike Thole

Sarah Wedde Alexander Kather

(Hrsg.)

Über die Notwendigkeit der Historischen Bildungsforschung

Wegbegleiter*innenschrift für Edith Glaser 


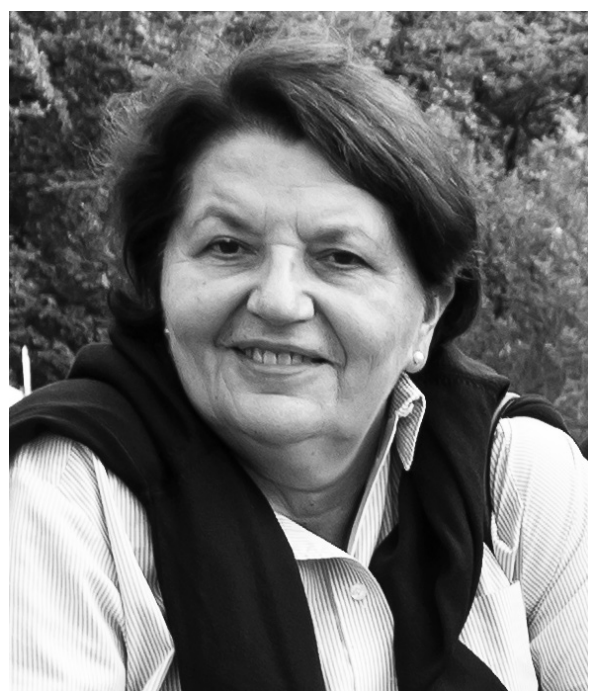

Edith Glaser ist seit 2006 Professorin für Historische Bildungsforschung an der Universität Kassel. Ihre Forschungsschwerpunkte sind die Disziplingeschichte der Erziehungswissenschaft, die historische Frauenund Geschlechterforschung sowie die geschichtliche Perspektive auf Bildungspolitik und Bildungsberatung. 


\section{Friederike Thole Sarah Wedde Alexander Kather (Hrsg.)}

\section{Über die Notwendigkeit der Historischen Bildungsforschung}

Wegbegleiter*innenschrift für Edith Glaser 
Dieser Titel wurde in das Programm des Verlages mittels eines Peer-Review-Verfahrens aufgenommen. Für weitere Informationen siehe www.klinkhardt.de.

Bibliografische Information der Deutschen Nationalbibliothek Die Deutsche Nationalbibliothek verzeichnet diese Publikation in der Deutschen Nationalbibliografie; detaillierte bibliografische Daten sind im Internet abrufbar über http://dnb.d-nb.de.

2021.n. () by Julius Klinkhardt.

Foto Umschlagseite 1: () Achim Heinrichs.

Druck und Bindung: AZ Druck und Datentechnik, Kempten.

Printed in Germany 2021.

Gedruckt auf chlorfrei gebleichtem alterungsbeständigem Papier.

(c) (i)(2) Die Publikation (mit Ausnahme aller Fotos, Grafiken und Abbildungen) ist veröffent-

licht unter der Creative Commons-Lizenz: CC BY-NC-SA 4.0 International

https://creativecommons.org/licenses/by-nc-sa/4.0/

ISBN 978-3-7815-5914-1 digital doi.org/10.35468/5914

ISBN 978-3-7815-2479-8 


\section{Inhaltsverzeichnis}

Friederike Thole, Sarah Wedde und Alexander Kather

Über die Notwendigkeit der Historischen Bildungsforschung -

Einführung in die Wegbegleiter*innenschrift für Edith Glaser

\section{Teil I}

Einblicke in ausgewählte Arbeitsbereiche der Historischen Bildungsforschung

Sarah Wedde und Friederike Thole

Historische Bildungsforschung schreibt Wissensgeschichte

\section{Ulrich Herrmann}

Die Bedeutung des Gemeinten im Gesagten.

Das Werkzeug des Historikers: Philologie und Hermeneutik -

am Beispiel von Wilhelm Flitners Veröffentlichungen in der NS-Zeit

Christian Bluhm und Markus Wochnik

Gender in der beruflichen Bildung - Entwicklung einer „Kategorie“

und einer zeitgemäßen Auseinandersetzung

\section{Pia Schmid}

Schule, Lehrerinnen und Lehrer in Autobiographien

politisch engagierter Frauen - 1850 bis 1910

Alexander Kather

„Wo die Quelle des Wissens ununterbrochen fließt“ -

Auslandsaufenthalte von Fremdsprachenlehrenden

aus (fach-)wissensgeschichtlicher Perspektive

Elke Kleinau

Children Born of War in der deutschen Nachkriegsgesellschaft -

Pädagogischer Diskurs und biografische Erzählungen 


\section{Teil II}

\section{Historische Bildungsforschung im inner- und interdisziplinären Kontext}

Ralf Mayer

,Zur Aufgabe des Erinnerns ‘ - im Spannungsfeld

von Orientierungsanspruch und Erinnerung

Werner Thole und Max-Ferdinand Zeterberg

Entdecken, Vergessen und Erinnern.

Über das ambivalente Rendezvous der Erziehungswissenschaft mit

der Sozialpädagogik bei Klaus Mollenhauer

Bernd Overwien

Wie politisch ist politische Bildung?

Kontroversen in der politischen Bildung seit den siebziger Jahren

Annedore Prengel

Anna und Maria mit dem Buch -

eine imaginierte pädagogische Beziehung

Friederike Heinzel und Julian Storck-Odabasi

100 Jahre Grundschule - Konzepte des Anfangsunterrichts

\section{Isabelle Naumann}

Von der Meritentafel zur Verhaltensampel -

Lob und Strafe in der Grundschule

Winfried Speitkamp

Vom Bauhaus lernen:

Das Staatliche Bauhaus von 1919 und die Universität der Zukunft 161

Verzeichnis der Autor*innen 


\section{Winfried Speitkamp}

\section{Vom Bauhaus lernen: Das Staatliche Bauhaus von 1919 und die Universität der Zukunft}

\section{Die Universität der Krise}

Kaum ein Bereich des öffentlichen Lebens wird durch die Corona-Krise der Gegenwart so in Frage gestellt wie die Universitäten. Handel und Dienstleistungsgewerbe werden nach der Krise weitgehend in den alten Arbeitsmodus zurückkehren; der Trend zum Online-Handel hat schon lange vor der Pandemie begonnen, er hätte sich auch ohne die Pandemie weiter verstärkt. Kultur und Sport setzen darauf, sich wieder für Publikum und Mitwirkende zu öffnen; eine Alternative ist nicht gewünscht und kaum möglich, so attraktiv die zusätzlichen Möglichkeiten beispielsweise digitaler Museumsbesuche sein mögen. Auch das Schulwesen wartet auf die Wiederkehr des regulären Präsenzunterrichts: Ob Grundschule, weiterführende Schule oder Berufsschule - alle betonen die Notwendigkeit der analogen Begegnung und des gemeinsamen Lernens am physischen Ort. Die Rückkehr zur „alten Normalität“ erscheint unvermeidlich und gewünscht.

Nicht so eindeutig ist die Perspektive der Hochschulen. Auch hier klaffen zwar die Einschätzungen zur aktuellen Lage weit auseinander: Während die einen programmatisch die Rückkehr zur Präsenzuniversität fordern und den Hochschulleitungen unterstellen, klammheimlich als kostengünstigere Variante der Hochschullehre den digitalen Unterricht auf Dauer einführen zu wollen, also eine zumindest hybride Hochschule vorzubereiten, betonen die anderen die Vorteile digitaler Vermittlungsformen, des asynchronen Lernens, der flexiblen und kreativen Nutzung buchstäblich grenzenloser digitaler Lern- und Kommunikationsräume. Und tatsächlich expandieren derzeit auch unabhängig von Corona gerade diejenigen Hochschulen, die ganz auf Fernlehre setzen: Die größte deutsche Hochschule ist mit über 70.000 Studierenden eine Fernuniversität, und in absehbarer Zeit wird sie sich diesen Rang mit einer weiteren - privaten - Hochschule teilen müssen, die sich ebenfalls auf Fernlehre konzentriert und über englischsprachige - kostenpflichtige - Studiengänge ein scheinbar unbegrenztes internationales Publikum anspricht. Derweil kämpfen die deutschen Präsenzhochschulen um Studieninteressierte und können angesichts der demographischen Entwicklung und der vielfältigen analogen und digitalen Studienangebote in aller Welt nicht einmal mehr sicher sein, deutsche Schulabsolvent*innen mehrheitlich auf Dauer an sich zu binden. 
Ungeachtet dessen scheinen viele Hochschulen in Deutschland nach wie vor auf eine Rückkehr zum Status quo ante zu setzen: auf eine Präsenzuniversität, auf die sogenannte "Massenuniversität", die den großen Bedarf an Nachwuchs etwa in den Bereichen Ingenieurwesen, Lehramt, Justiz oder Medizin durch Großveranstaltungen, auch durch Vorlesungen mit Hunderten von Studierenden deckt und die Lehre mit entsprechenden Prüfungsformen, sogar analogen Massenklausuren, verbindet. Selbst zum bisherigen Höhepunkt der Corona-Krise haben die meisten Hochschulen zwar weitgehend auf Präsenzvorlesungen, nicht aber im gleichen Maß auf Präsenzprüfungen verzichtet. Dennoch dämmert es mittlerweile vielen Verantwortlichen in den Hochschulen, dass eine Rückkehr zur „alten Normalität" kaum mehr vorstellbar ist, dass aber der Rahmen und die Grundzüge einer „neuen Normalität“ noch nicht erkennbar sind. Gesucht wird nach einem neuen Modell der Hochschule - und dies mitten in einer Krise, deren Ausgang ungewiss ist. Das hängt zum einen damit zusammen, dass die Vorstellung, künftig wieder Hunderte Studierende in einem engen Hörsaal zusammenzubringen, auf absehbare Zeit nicht realistisch erscheint. Zum anderen steht das Format der Vorlesung - als mehr oder minder interaktive, oft jedenfalls immer noch eher frontale Präsentation von Lehrenden vor einem vornehmlich rezipierenden Massenpublikum und mit einer Klausur am Ende - schon seit Jahrzehnten in der Kritik. Digitale Lehr- und Prüfungsformen, die durch die Corona-Krise einen Schub erhalten haben, bieten nunmehr eine Fülle von effektiveren Varianten des Lernens und der Lernkontrolle an.

Gefragt wird also nach dem Bildungsraum der Zukunft, nach einem neuen Modell der Universität. Die Fernuniversität als generelle Lösung kann es kaum sein: Erstens müssten Studierende nicht digital in Paderborn, Passau oder Kassel studieren, wenn ohne zusätzliche Mühe und Kosten ein digitales Studium an deutschen Exzellenz-Universitäten möglich wäre. Zweitens könnte, wer Gebühren in Kauf nimmt, doch auch einen Abschluss von renommierten internationalen Universitäten wie Oxford oder Harvard erlangen, ohne dort eine aufwendige und teure Präsenzphase einzulegen. Und drittens expandieren, wie oben angesprochen, mittlerweile die kostenfreien ebenso wie die kostenpflichtigen digitalen Studienangebote derjenigen deutschen staatlichen und privaten Hochschulen, die sich bereits darauf spezialisiert haben und dank ihres Startvorsprungs ein weitgefächertes, professionell betreutes Fernstudium ermöglichen. Wie also kann - in der Krise, aber nicht nur wegen der Krise - Neues entstehen, die Universität neu gedacht werden? Die „Suche nach der neuen Universität“ (Mälzer, 2016) ist nicht grundsätzlich neu, sondern reicht bis ins 19. Jahrhundert zurück. Das zeigt auch der Blick auf das historische Bauhaus, das als Ausgangspunkt der folgenden Überlegungen dient. Von da wird der Bogen geschlagen über die jüngere Initiative, ein neues Europäisches Bauhaus zu gründen, hin zu Gedanken über die Universität der Zukunft. 


\section{Das Weimarer Bauhaus von 1919}

Im April 1919 wurde in Weimar das Staatliche Bauhaus gegründet. Unter der Leitung des Architekten Walter Gropius entstand eine Gestaltungsschule, die dem ersten Anschein nach alles neu machte, anders jedenfalls als bisherige Kunstschulen - so wurde es häufig noch beim Bauhaus-Jubiläum von 2019 vermittelt. Tatsächlich allerdings stand auch das Bauhaus von 1919 in einer Tradition: 1860 war in Weimar die Großherzoglich-Sächsische Kunstschule eingerichtet worden, 1908 eine Kunstgewerbeschule. Sie wurde bis 1915 von dem belgischen Architekten und Designer Henry van de Velde geleitet, der im Ersten Weltkrieg Deutschland verlassen musste. Als Nachfolger schlug er Walter Gropius vor - aber nicht erst Gropius, sondern schon van de Velde leitete in seinem Schaffen, auch wenn es dem Jugendstil verhaftet blieb, bereits den Übergang zur Sachlichkeit ein.

Van de Velde und Gropius gehörten beide dem 1907 ins Leben gerufenen Deutschen Werkbund an. Dort trafen sich Bildungs- und Wirtschaftsbürger, Künstler, Industrielle und Politiker, um über die Zukunft der Industriegesellschaft nachzudenken, Ästhetik, Tradition, Kunstgewerbe, Handwerk und industrielle Produktion wieder zusammenzuführen und eine Veredelung der Massenproduktion einzuleiten. Auch Friedrich Naumann, Theologe und liberaler Politiker des späten Kaiserreichs, gehörte dazu. Naumann war bis zu seinem frühen Tod am 24. August 1919 Mitglied der Weimarer Nationalversammlung und hatte noch Einfluss auf deren Verfassungsberatungen. Die Weimarer Reichsverfassung vom 11. August 1919 spiegelte den Zeitgeist der Sachlichkeit und Nüchternheit, etwa im Verzicht auf Adel, Orden und Ehrenzeichen sowie in der Schlichtheit der Staatssymbolik. Gerade dieser Geist der Nüchternheit, jedenfalls der Verzicht auf Ornamentik und Historismus, wurde auch dem Bauhaus zugeschrieben. Nur war all dies nicht, wie der Mythos bis heute zu suggerieren versucht, ein gänzlicher Neuanfang, sondern schöpfte aus der Erfahrung und dem Austausch im späten Kaiserreich.

Das gilt auch für das Bildungsideal. Dieses zielte nicht allein auf die Distanzierung von der elitären Kunstausbildung in den Akademien des 19. Jahrhunderts. In dieser Hinsicht ging das Bauhaus tatsächlich konsequent und demonstrativ vor auch wenn es sich beim Vormodernen bediente: Der Begriff „Bauhaus“ war den Bauhütten des Mittelalters entlehnt, und die Lehrer arbeiteten am Bauhaus als „Meister“, die Lehrerschaft firmierte als „Meisterrat“. Die Studierenden starteten als Lehrlinge und konnten dann zu "Gesellen“ und "Jungmeistern“ aufsteigen - so das Prinzip. In Dessau, wohin das Bauhaus 1925 aus politischen Gründen ausweichen musste, wurde diese Begrifflichkeit allerdings aufgegeben. Ungewöhnlich und fast revolutionär war das Geschlechterverhältnis unter den Studierenden: Von den im Sommer 1919 aufgenommenen 163 Studierenden waren 79 männlich und 84 weiblich. Die Gesamtzahl wurde im Übrigen nie erheblich größer, auch am Ende waren es nur jeweils 150 bis 200 junge Männer und Frauen, die am 
Bauhaus studierten. Das Lehrpersonal, obgleich fast durchweg männlich, war recht heterogen und zudem international. $\mathrm{Zu}$ den Lehrern zählten mit Laszlo Moholy-Nagy, Oskar Schlemmer, Paul Klee, Wassily Kandinsky, Gerhard Marcks, Lyonel Feininger und Josef Albers viele der bedeutendsten Künstler und Gestalter der Zeit. Die Textildesignerin Gunta Stölzl war eine der wenigen lehrenden Frauen am Bauhaus. Zum Lehrpersonal gehörte auch der Schweizer Maler und Kunstpädagoge Johannes Itten, der mit seiner Farbenlehre, die Formen und Farben zuordnete und abstufte, Einfluss in der Kunsttheorie gewann. Itten stand der Ende des 19. Jahrhunderts aufgekommenen Mazdaznan-Gemeinschaft nahe, einer synkretistischen, von fernöstlichen Religionen inspirierten, in manchen Zügen rassistischen Gruppierung. 1923 schied er im Dissens mit Gropius aus dem Bauhaus aus; er spiegelte die Diversität der Ideen und Konzepte, die anfangs am Bauhaus zusammenkamen, wider.

Gemeinsam war den Bauhaus-Ideen der wiederum in dem Aufbruch der Epoche um 1900 wurzelnde reformpädagogische, ganzheitliche Ansatz, die Suche nach einer Verbindung von Körper und Geist, handwerklich-künstlerischer Ausbildung und intellektueller Reifung. Alle Studierenden des frühen Bauhauses mussten einen Vorkurs durchlaufen, in dem der Umgang mit Materialien und Handwerkstechniken gelehrt wurde. Zur handwerklichen Ausbildung sollte eine zeichnerische und malerische sowie eine wissenschaftlich-theoretische Ausbildung treten. Abgesehen von der handwerklichen Ausbildung mit der Meisterprüfung und dem Abschluss in der Baulehre konnte man aber am Bauhaus vorerst keine Abschlüsse erwerben; ein eigenes Bauhaus-Diplom gab es erst ab 1926.

Gropius' Ideen kulminierten in der Vision eines Gesamtkunstwerks, das es zu erschaffen gelte. Das von ihm im April 1919 entworfene Programm des Staatlichen Bauhauses formulierte: „Das Bauhaus erstrebt die Sammlung alles künstlerischen Schaffens zur Einheit, die Wiedervereinigung aller werkkünstlerischen Disziplinen - Bildhauerei, Malerei, Kunstgewerbe und Handwerk - zu einer neuen Baukunst als deren unablösliche Bestandteile. Das letzte, wenn auch ferne Ziel des Bauhauses ist das Einheitskunstwerk - der große Bau -, in dem es keine Grenze gibt zwischen monumentaler und dekorativer Kunst." Das Programm endete in den emphatischen Worten: „Wollen, erdenken, erschaffen wir gemeinsam den neuen Bau der Zukunft, der alles in einer Gestalt sein wird: Architektur und Plastik und Malerei, der aus Millionen Händen der Handwerker einst gen Himmel steigen wird als kristallenes Sinnbild eines neuen kommenden Glaubens." (Programmschrift, 1919/2009)

Der Glaube an einen neuen Glauben, das Gesamtkunstwerk als Ziel, das Klassenübergreifende, die Verbindung zum Handwerk - später die Verbindung zur Technik („Kunst und Technik - eine neue Einheit“, proklamierte Gropius ab 1923) - all das war mehr als nur eine Reform der Ausbildung, das zielte, in der Tradition von Reformpädagogik und Lebensreform um 1900, auf eine neue Ge- 
sellschaft und schließlich auf einen ,Neuen Menschen'. Harry Graf Kessler war überrascht, als er im Juli 1919 das drei Monate zuvor gegründete Staatliche Bauhaus in Weimar besuchte, denn dort schien es ihm nicht vorrangig um Kunst und Gestaltung zu gehen, sondern um Lebensreform: „Sie suchen [...] vor allem den neuen Menschen", notierte er in seinem Tagebuch (zit. n. Wilhelm, 1994, S. 68). Ähnlich nahm es Oskar Schlemmer wahr, der selbst von 1920 bis 1929 am Bauhaus lehrte. Dort baue man "nach ganz anderer Seite hin [...] als erwartet wird, nämlich: den Menschen" (ebd.). Die Selbstsicherheit, in Weimar eine neue Gesellschaft, eine neue Lebensform und einen ,Neuen Menschen' quasi aus einem Guss entstehen zu lassen, diese konsequent anthropozentrische Weltsicht, führte freilich zur Selbstüberschätzung, die durch das von Walter Gropius betriebene geschickte Marketing noch gestärkt wurde und bis heute den Mythos Bauhaus befeuert - selbst die im Zuge des Bauhaus-Jubiläums 2019 geführten kritischen Debatten etwa in Bezug auf die Rolle von Frauen am Bauhaus konnten dem Mythos wenig anhaben.

Dabei hatte das Bauhaus keineswegs alles neu gemacht und es stand auch keineswegs allein da. Es gab Vorläufer, es gab parallele Entwicklungen und es kam zu zahlreichen Folgegründungen, die eigenständigen Charakter gewannen. Die Gestaltungsmoderne entstand im Werkbund, zu sehen 1914 in der Werkbundausstellung in Köln, wo Gestaltung und Architektur zusammen gedacht wurden, ebenso in Darmstadt und dem Jugendstilaufbruch. Gropius und der letzte Bauhaus-Direktor Ludwig Mies van der Rohe hatten vor dem Ersten Weltkrieg im Atelier des Architekten Peter Behrens gelernt, der ebenfalls Mitglied im Deutschen Werkbund war und in Darmstadt auf der Mathildenhöhe gebaut hatte. In der Darmstädter Bauausstellung von 1901 auf der Mathildenhöhe wurde die Verbindung von Handwerk, Kunst, Architektur und Lebensreform lange vor dem Bauhaus gedacht und gezeigt. Der Jugendstil-Architekt Joseph Maria Olbrich gestaltete die Ausstellungsarchitektur und formulierte den das Bauhaus vorwegnehmenden Anspruch: „Eine Stadt müssen wir erbauen, eine ganze Stadt. Alles andere ist nichts." (Asshoff \& Verhoeven, 2017)

Und es gab zahlreiche parallele Reformschulen und Gestaltungsansätze in Deutschland und weltweit. Dazu gehörten die Vereinigung von Kunstakademie und Kunstgewerbeschule in Düsseldorf 1919, die „Kölner Progressiven“ (eine Künstlergruppe der 1920er Jahre) und die Kalltall-Gemeinschaft in der Eifel, die soziales Leben und künstlerische Darstellung verbinden wollte, die „Schule der Weisheit" in Darmstadt, in Frankfurt am Main das Jüdische Lehrhaus von Franz Rosenzweig, die Burg Giebichenstein, also die 1915 gegründete, ebenfalls auf Werkbundideen aufbauende Gestaltungsschule in Halle, und schließlich Wchuchtemas, die 1920 gegründete, 1930 aufgelöste avantgardistische Kunsthochschule in Moskau. Sie alle strebten - in Varianten - eine ganzheitliche Reform und einen ,Neuen Menschen' an und wollten wieder Hoffnung und Mut 
machen zur Gestaltung der Gegenwart und der Gesellschaft. Indes entwickelten besonders das Bauhaus und hier vor allem Gropius eine ungeheure Kraft der Monopolisierung und Kanonisierung der Moderne.

Seit 1933 strahlte das Bauhaus seinerseits in die Welt aus, getragen auch durch die Emigration vieler Mitglieder und der ehemaligen Direktoren Walter Gropius und Ludwig Mies van der Rohe, die als Architekten in den USA arbeiteten. Lazlo Moholy-Nagy leitete in Chicago das New Bauhaus als private amerikanische Nachfolgeinstitution. Vielleicht am bemerkenswertesten unter den Folgegründungen ist das 1933 gegründete, bis 1957 bestehende Black Mountain College in North Carolina, wo unter anderem das ehedem am Bauhaus tätige Ehepaar Josef und Anni Albers, Maler der eine, Textilkünstlerin die andere, wirkten; Josef Albers amtierte 1948/49 auch als Rektor des College. Bei dieser Schule handelte es sich um eine besondere Aneignung des Bauhaus-Erbes, denn hier traten zu den Gestaltungsdisziplinen auch Geistes-, Natur- und Wirtschaftswissenschaften und man strebte einen tatsächlich umfassenden, ganzheitlichen Zugang zu den Aufgaben der Gegenwart an. Noch nach dem Zweiten Weltkrieg kam es zu Folgegründungen, so zum Beispiel 1955 mit der Hochschule für Gestaltung Ulm unter dem Schweizer Architekten Max Bill als Rektor, der seinerseits am Bauhaus studiert hatte. Die Ulmer Hochschule wurde 1968 wieder geschlossen. Nur die wenigsten Einrichtungen überlebten, viele gingen unter - aus politischen, materiellen oder personellen Gründen. Die Ideen, Gestaltungsansätze, Bauvorstellungen und Stadtmodelle des Bauhauses wirkten indes weiter, bis hinein in popularisierte und standardisierte Formen der Architektur und des Designs der Gegenwart. Auch die Postmoderne hat dem nichts anhaben können. Die Ausstrahlung des Bauhauses bis heute basiert auf der Migration von Menschen, Ideen, Formen und Stilen. Dabei wurde das Vorbild überall neu adaptiert, weiterentwickelt, angepasst und integriert, globalisiert und glokalisiert. Der Mythos Bauhaus lebt in dieser Wanderungsgeschichte, die eine Transfer- und Transformationsgeschichte ist, fort.

\section{Das neue Europäische Bauhaus}

$\mathrm{Zu}$ diesen manchmal verblüffenden Adaptionsprozessen gehört auch das neue Europäische Bauhaus, von dem jüngst die Rede ist. Die Präsidentin der Europäischen Kommission Ursula von der Leyen hielt am 16. September 2020 eine Rede zur Lage der Europäischen Union, in der sie ihr Programm des Green Deal erläuterte, die Bedeutung von Nachhaltigkeit im Bauen unterstrich und eine Renovierungswelle für ganz Europa ankündigte. An eher versteckter Stelle verwies sie dabei auf das Bauhaus von 1919, und zwar in einer ganz besonderen Beziehung: Schon das Bauhaus habe Bauen und Architektur einerseits, Gestaltung und Ästhe- 
tik andererseits zusammengedacht und zusammengebracht. Es gehe nun darum, den Ansatz des Bauhauses auf die Herausforderungen der Gegenwart anzuwenden und Nachhaltigkeit mit Ästhetik zu verbinden.

Diesen Gedanken vertiefte und variierte sie in den folgenden Wochen in weiteren Erklärungen und Artikeln. Das Projekt ist beständig in Bewegung und wird weiterentwickelt. Immer wieder unterstrichen wird nun die Notwendigkeit, Nachhaltigkeit, Innovation, Technik und Gestaltung zusammen zu denken und zu bringen. Wie beim historischen Bauhaus sollen Ingenieurwesen, Wissenschaft, Architektur, Planung, Kunst, Gestaltung und Kultur gemeinsam daran arbeiten, gute, sozial gerechte, nachhaltige, ansprechende und akzeptierte Konzepte für die gesamte Gesellschaft zu erarbeiten. Wiederum wird die Notwendigkeit beschworen, interdisziplinär zu denken und zu arbeiten, dabei Experiment und Kreativität $\mathrm{zu}$ vereinen. Bei alledem gehe es darum zu ermitteln, wie Leben und Wohnen künftig gestaltet werden könnten. Ganz konkrete Lösungen im Blick auf die Verwendung neuer bzw. wiederentdeckter Werkstoffe wie Holz sollen angestrebt werden. Dieser Erneuerungsprozess soll von einem grundlegenden Prozess der Digitalisierung begleitet und getragen werden; Potentiale des digitalen Entwurfs, der digitalen Simulation und Planung bis hin zur digital unterstützten Produktion sind hier eingeschlossen.

Kern der Initiative war und ist die Ankündigung, in fünf Staaten der Europäischen Union im Jahr 2022 fünf neue europäische Bauhäuser zu gründen, in denen spezifische Fragen der Nachhaltigkeit, der CO2-Reduktion, des Klimawandels oder des künftigen Bauens behandelt werden sollen; weitere Bauhäuser in anderen europäischen Staaten sollen folgen. In einem Design-Prozess sollen vorerst Ideen aus ganz Europa gesammelt werden; dabei, so das Brüsseler Postulat, soll das neue Konzept der Europa-Bauhäuser in einem partizipativen Bottom-Up-Prozess entwickelt werden und dann in einem Wettbewerb zu innovativen Umsetzungen führen. Konkrete Erläuterungen, was unter den neuen Europäischen Bauhäusern verstanden werden könnte, stehen vorerst aus - dies mit Hinweis auf Offenheit und Breite eines gesamteuropäischen Design-Prozesses. Erneut erscheint das Bauhaus als offener Raum für Ideen und Aushandlungen, nicht als fixiertes Programm, schon gar nicht als feste Schule.

Die Initiative der Kommissionspräsidentin von der Leyen rief nicht nur Zustimmung hervor, sie provozierte auch Kritik. Das reichte von der Frage, ob das Bauhaus, ein deutsches und zeitspezifisches Phänomen der 1920er Jahre, überhaupt als Vorbild für die Fragen der Gegenwart geeignet sei, bis zur Kritik an einem eurozentrischen Lösungsansatz, am Festhalten an einem ökonomischen Wachstumsmodell, während die sozialen und humanitären Defizite der Europäischen Union etwa im Blick auf die globalen Fluchtbewegungen und die Abschottung an den Grenzen Europas übergangen würden. Doch ungeachtet dieser anfangs geäußerten Einwände elektrisiert die Initiative von der Leyens vielfältige Ak- 
teur*innen in Städten, Universitäten und anderen Institutionen, die sich mit Themen der Nachhaltigkeit und des Klimawandels befassen. Zum ersten Mal seit längerer Zeit scheint die vermeintlich kalt-bürokratische Europäische Union ein auch emotional mobilisierendes Gemeinschaftsprojekt gefunden zu haben, das nun selbst Kritiker*innen am Ende zum Mittun animiert. Auf der Website der Europäischen Union können beispielhafte Projekte eingestellt werden, ein erster Wettbewerb und die Ausschreibung von Forschungs- und Innovationsprojekten sind angekündigt. In zahlreichen Staaten der Europäischen Union beginnen - oft unter regierungsamtlicher Federführung - Planungen für die Bewerbung um ein Europäisches Bauhaus; wieder einmal, wie schon beim Bauhaus-Jubiläum 2019, erheben viele nun den Anspruch, den Geist und den Kern des Bauhauses zu repräsentieren. Die derzeitigen Initiativen umfassen sehr konkrete, anwendungsorientierte Projekte zur Umgestaltung der Städte und zum nachhaltigen Umgang mit Ressourcen in der Architektur, aber auch Konzepte virtueller Plattformen des Austauschs und der Kooperation und erstrecken sich zudem ebenso auf das ideelle und pädagogische Erbe des Bauhauses.

Trotz der Vielfalt der Ansätze - und auch trotz der schnell aufbrechenden Konkurrenz zwischen Staaten, Bundesländern, Städten und Hochschulen - zeigt sich dabei, wie lebendig Kernideen und Ansätze des Bauhauses nach wie vor sind. So scheint erstens breite Übereinstimmung zu herrschen, dass allein ein interdisziplinäres Vorgehen beitragen kann, die aktuellen Herausforderungen in Bezug auf Klima und Nachhaltigkeit zu lösen, und zwar unter gleichberechtigter Einbeziehung von Gestaltung, Kunst und Ästhetik, und diese nicht verstanden als Elemente einer bloß äußerlichen Verschönerung, sondern als Teil einer nachhaltigen Formgebung künftiger Lebensweisen. Zweitens findet offenbar Aufmerksamkeit, dass im Begriff des Bauhauses ein pädagogischer Ansatz vorborgen ist. Das Bauhaus war eben eine Schule, an der nicht nur herausragende zeitgenössische Gestalter*innen tätig waren, sondern die vor allem künftige Gestalter*innen ausbildete und bildete, also nachhaltig und generationenübergreifend wirken wollte. Darin liegt eine aktuelle Herausforderung, denn die Kritik an der Gedankenlosigkeit der Gesellschaft und der Tatenlosigkeit der aktuellen politischen Akteure - ausgedrückt in der Person Greta Thunberg und der „Fridays for Future“-Bewegung - spiegelt einen Generationenkonflikt, der offenbar erneut eine pädagogische und partizipative Lösung erfordert. Und drittens scheint sich in der Begeisterung für die neue Initiative zu einem Europäischen Bauhaus doch die Hoffnung auf eine inklusive Lösung für die gesamte Gesellschaft auszudrücken. Auch die neue Klima-Jugendbewegung wird getragen von der Sehnsucht nach einer gesellschaftlichen Umkehr und nach einer ganzheitlichen Erlösung der Menschheit. Die ideell überholt erscheinende und jedenfalls politisch desavouierte Vision eines Gesamtkunstwerks und eines ,Neuen Menschen' ist immer noch und wieder virulent. Damit muss sorgsam umgehen, wer künftige Generationen unterrichten will. 


\section{Die Universität der Zukunft}

Für Universitäten ist dies eine fundamentale Herausforderung. Man kann Ihnen zwar kaum noch vorwerfen, dass hier in Elfenbeintürmen weltabgewandt realitätsferne Wissenschaft betrieben würde, aber sie wählen doch unterschiedliche Wege bei der Frage, wie die Verbindung von Forschung und Lehre mit tagesaktuellen Aufgaben gefunden und ausgestaltet wird. Dies bündelt sich im Begriff des Transfers, den manche als „Dritte Mission“ der Universitäten verstehen. Transfer meint - weit gefasst - drei Bereiche, nämlich Kommunikation, Intervention und Innovation: Kommunikation in und mit der Gesellschaft, was wissenschaftliche Erkenntnisse und Herangehensweisen angeht; Intervention in gesellschaftliche Prozesse etwa durch Stellungnahmen, Ausstellungen und Beratungsangebote; Innovation durch die Anwendung und Umsetzung von Erfindungen und Erkenntnissen im öffentlichen Bereich, in Wirtschaft und Gesellschaft.

Eine Universität der Zukunft kann dies nicht als zusätzliches, gesondert administriertes Betätigungsfeld ansehen, sondern muss es mit den hergebrachten Kernaufgaben Forschung und Lehre (und auch Lernen) verbinden. Universitäten bilden diejenigen aus, die künftig Entscheidungen treffen und verantworten werden. Sie müssen Wissen und Kompetenzen vermitteln, die künftige Generationen befähigen, ihre Gesellschaft immer neu zu gestalten. Angesichts der Unüberschaubarkeit und Dynamik des Wissens, das zu bewältigen ist, müssen Universitäten besonders die Kompetenz vermitteln, gerade diejenigen Situationen und Herausforderungen zu bewältigen, die man noch nicht kennen oder auch nur antizipieren kann. Das haben einerseits die großen Krisen der Gegenwart (Klima und Corona) deutlich gemacht, andererseits aber die jüngeren Umbrüche in Wirtschaft, Gesellschaft und Umwelt, die oft durch kontingent erscheinende Ereignisse (z.B. Bankenkrise seit 2007 und Eurokrise seit 2010, Terroranschläge vom 11. September 2001, Reaktorunfall in Fukushima vom März 2011) fühlbar geworden sind. Studierende müssen lernen, dass ihr Wissen schneller als je zuvor veralten wird, dass sich voraussichtlich auch ihr Berufsfeld im Laufe eines Arbeitslebens von mehreren Jahrzehnten mehrfach ändern wird. Universitäten müssen lernen, Studierende genau darauf vorzubereiten. Sie müssen lehren, ins Offene, ins Ungewisse zu leben, zu denken und zu planen. Dabei können sie auch vom Bauhaus lernen.

Dazu gehört die Bereitschaft, sich auch in existenzbedrohenden Krisen nicht auf die bloße Stabilisierung des Erreichten zurückzuziehen, sondern Unerwartetes zu erwarten, sich an Ungewohntes zu gewöhnen, dafür neue Wege zu suchen. Anstelle des Verharrens in tradierten Arbeitsstrukturen, Aufgabenfeldern und Lernsystemen benötigt es dazu Temperament, Kreativität und Innovationskraft. Kreativität entsteht vor allem durch eine Begegnung mit Anderem, mit Fremdem. Das Bauhaus hat solche Begegnungen provoziert, hat Inter- und Transdisziplinarität erprobt, bevor diese Begriffe bekannt waren. Die unüberschaubaren Brüche der 
deutschen Nachkriegszeit von 1919 suchte man durch eine neuartige Zusammenführung von Denk-, Arbeits- und Gestaltungsweisen zu überwinden. Die Fragen der Zukunft waren dabei durchaus ungewiss. Zukunftsprognosen gab es viele, zu viele, konkurrierende - Utopien ebenso wie Dystopien. Auch heute können Studierende auf Herausforderungen der Zukunft nur in multiperspektivischen Herangehensweisen vorbereitet werden. Jüngere Tendenzen in der Lehre spiegeln das bereits. Zahlreiche neuere Studiengänge sind interdisziplinär angelegt, verbinden etwa Ökonomie und Philosophie, Wirtschaft und Technik oder Sprachen und Betriebswirtschaft. Erst recht verspricht die Verknüpfung von Wissenschaft und Gestaltung neue Möglichkeiten, Bildung zu denken und zu erleben. Es geht darum, endliche Ressourcen, technische Aufgaben und gestalterische Lösungen zusammenzubringen. Es geht um die Formgebung der Zukunft.

In dieser Perspektive können Universitäten sich wieder - und in gewisser Hinsicht durchaus im Aufgreifen auch Humboldtscher Ansätze - an erster Stelle als Bildungs- und Lernorte verstehen, als Orte, in denen Menschen zusammenkommen, um miteinander und voneinander zu lernen. Kreativität, interdisziplinäre Offenheit und Verständnis für heterogene Arbeits- und Denkweisen sind dabei Voraussetzung und Lernziel zugleich. Es geht dabei nicht nur darum, was gelehrt und gelernt werden soll, sondern vor allem darum, wie gelernt wird. Lernen als zu gestaltender Prozess und als Prozess der Teilhabe erfordert konkrete neue Angebote, Räume der Begegnung und des Austauschs, ob man sie nun als Denkwerkstätten, Lernlabore (die hier nicht als fachdidaktische Übungsräume verstanden werden) oder Sense Labs bezeichnet und ausgestaltet. Prototypisch findet man sie bereits an manchen nordamerikanischen Universitäten. Ein solches Lernen setzt Durchlässigkeit, die Möglichkeit zu Aus- und Umstieg, die Auflösung versäulter Strukturen voraus. Das schließt die Nutzung digitaler Instrumente und Optionen ein, etwa im Sinne hybriden Arbeitens, asynchronen Lernens und der virtuellen Modellierung und Simulation. Der Prozess des Lernens wird sich dadurch öffnen, denn Lernen bleibt ein individueller, letztlich nur begrenzt zu steuernder, vielmehr vor allem zu ermöglichender Prozess der Aneignung und Adaption; Lehrende können nicht programmieren, was Lernende lernen.

Eine Massenbeschulung an Universitäten, wie sie mit den Bologna-Reformen verbunden scheint, aber schon vorher beklagt worden ist, sollte dann freilich vorbei sein. Große Vorlesungen können ins Digitale verlagert werden. Große Hörsäle mit festen Bestuhlungen zum Beispiel sind überholt - die Corona-Krise hat auch die bauliche Fehlentwicklung an den Universitäten während der letzten Jahrzehnte offengelegt. Die Universität der Zukunft benötigt modulartig aufgebaute, insofern flexibel zu variierende Lehr-, Lern- und Forschungsräume. Sie wird in kleineren Einheiten arbeiten: kompakt, beweglich, transdisziplinär, hybrid, projektorientiert, kooperativ. Ihre Aufgabe ist es - wie am Bauhaus von 1919 - Menschen stark zu machen für das Kommende und Persönlichkeiten zu bilden, die 
auf die Herausforderungen der Zukunft reagieren können, die offen denken und verantwortungsbewusst handeln - Persönlichkeiten, die sich um Antworten auf die Fragen bemühen, die gegenwärtig zur Zerreißprobe der Gesellschaft zu werden drohen: Wie wollen wir leben? Wie können wir zusammenleben? Der Blick auf das Bauhaus, auf Vorgeschichte, Konzept und Praxis, auf Rezeption, Wirkung und Aktualität, zeigt den Ertrag historischer Forschung und kann das Bewusstsein offenhalten für die Transformation und Adaption von Ideen und Konzepten in der Bildungs- und Universitätsgeschichte.

\section{Literatur}

Asshoff, Carmen \& Verhoeven, Jennifer (Hrsg.) (2017). „Eine Stadt müssen wir erbauen, eine ganze Stadt!" Die Künstlerkolonie Darmstadt auf der Mathildenhöhe. Darmstadt: Theiss.

Baumhoff, Anja (2001). Das Bauhaus. In Etienne François \& Hagen Schulze (Hrsg.), Deutsche Erinnerungsorte. Bd. 2. München: C.H. Beck, S. 584-600.

Bundeszentrale für politische Bildung (2016). Der Neue Mensch. Aus Politik und Zeitgeschichte 65, S. 37-38.

Gerstner, Alexandra, Könczöl, Barbara \& Nentwig, Janina (Hrsg.) (2006). Der Neue Mensch. Utopien, Leitbilder und Reformkonzepte zwischen den Weltkriegen. Frankfurt a.M.: Peter Lang.

Gropius, Walter (1923). Idee und Aufbau des staatlichen Bauhauses. München: Bauhausverlag.

Gropius, Walter (1988). Ausgewählte Schriften. Berlin: Verlag für Bauwesen.

Kerbs, Diethart \& Reulecke, Jürgen (Hrsg.) (1998). Handbuch der deutschen Reformbewegungen 18801933. Wuppertal: Peter Hammer.

Küenzlen, Gottfried (1994). Der Neue Mensch. Zur säkularen Religionsgeschichte der Moderne. München: Wilhelm Fink Verlag.

Lepp, Nicola, Roth, Martin \& Vogel, Klaus (Hrsg.) (1999). Der Neue Mensch. Obsessionen des 20. Jahrhunderts. Ostfildern: Cantz.

Mälzer, Moritz (2016). Auf der Suche nach der neuen Universität: Die Entstehung der „Reformuniversitäten“ Konstanz und Bielefeld in den 1960er Jahren. Göttingen: Vandenhoeck \& Ruprecht.

Nerdinger, Winfried (2018). Das Bauhaus. Werkstatt der Moderne. München: C.H. Beck.

Nerdinger, Winfried (2019). Walter Gropius. Architekt der Moderne 1883-1969. München: C.H. Beck.

Programmschrift des Staatlichen Bauhauses in Weimar (1919/2009). Faksimiledruck des Thüringen Hauptstaatsarchivs Weimar. Weimar.

Schimpf, Cornelia (2008). Versagen einer Zufluchtsstadt. Kulturpolitik in der Weimarer Republik am Beispiel des Staatlichen Bauhauses in Weimar von 1919 bis 1925. Berlin: Lit Verlag.

Simon-Ritz, Frank, Winkler, Klaus-Jürgen \& Zimmermann, Gerd (Hrsg.) (2010). Aber wir sind! Wir wollen! Und wir schaffen! Von der Großherzoglichen Kunstschule zur Bauhaus-Universität Weimar, 1860-2010. Bd. 1. Weimar: Lit Verlag.

Speitkamp, Winfried (2019). Bauhaus neu denken. Zur Einführung. In ders. \& Claudia Weinreich (Hrsg.), Idee Inhalt Form. Beiträge zur Gestaltung der Gegenwart. Weimar: Bauhaus-Universität Weimar, S. 15-22.

Wilhelm, Karin (1994). Auf der Suche nach dem neuen Menschen. Zum Verhältnis von Walter Gropius und Johannes Itten. In Thomas Föhl (Hrsg.), Das frühe Bauhaus und Johannes Itten. Katalogbuch anläßlich des 75. Gründungsjubiläums des Staatlichen Bauhauses in Weimar. Ostfildern, S. 59-71. 